\title{
28 Research Suare \\ PD-1/PD-L1 Expressions on Treg Cells in Patients with Autoimmune Thyroid Disease
}

\section{Lin Cui}

Second Affiliated Hospital of Dalian Medical University

Xiaotong Gu

Second Affiliated Hospital of Dalian Medical University

Haixia Liu ( $\sim$ dllhx1017@163.com )

Second Affiliated Hospital of Dalian Medical University

Hong Zheng

Second Affiliated Hospital of Dalian Medical University

\section{Research Article}

Keywords: Autoimmune thyroid disease(AITD), Hashimotos'thyroiditis (HT), graves disease (GD)

Posted Date: August 18th, 2021

DOI: https://doi.org/10.21203/rs.3.rs-733027/v1

License: (c) (1) This work is licensed under a Creative Commons Attribution 4.0 International License. Read Full License 


\section{Abstract}

Background: Autoimmune thyroid disease(AITD) is a frequent autoimmune disease characterized by lymphocytic infiltration and thyroid autoantibody production caused by autoimmune dysfunction. Recent studies have shown that Treg cells can participate in the pathogenesis of AITD by inhibiting peripheral reactive $T$ cells and thereby inhibiting the autoimmune responses. Programmed cell death-1 (PD1)/programmed cell death ligand (PD-L1) pathway is a negative costimulatory pathway discovered in recent years, enhancing or blocking this pathway is associated with the immune process of AITD. PD1/PD-L1 was simultaneously expressed on Treg cells. In this paper, the role of regulatory T cells and PD1/PD-L1 signaling pathway in AITD was discussed.

Methods: We detected the percentage of CD4+CD25+CD127lowT cells, the ratio of PD-1+Treg cells and PD-L1+Treg cells in peripheral blood of twenty patients newly diagnosed with graves disease (GD) and twenty-one patients newly diagnosed with Hashimotos'thyroiditis (HT) patients by flow cytometry, and samples from twenty healthy individuals served as control.

Results: The results demonstrated that the proportion of CD4+CD25+CD127lowT cells in peripheral blood of $\mathrm{HT}$ patients was lower than that of healthy controls $(\mathrm{HCs})$, the ratio of PD-1+Treg cells of HT patients was higher than that of the HCs and GD patients, the ratio of PD-1+Treg cells of GD patients was higher than that of the HCs, the ratio of PD-L1+Treg cells in Treg cells of HT patients was higher than that of the HCs and GD patients and the ratio of PD-L1+Treg cells of GD patients was higher than that of the HCs. All the above were statistically significant.

Conclusions: The treg cells play an obvious role in hashimoto's thyroiditis, but not obvious in Graves' disease.

\section{Introduction}

Autoimmune thyroid disease (AITD), which encompasses two types: autoimmune hyperthyroidism known as Graves' disease (GD) and autoimmune Hashimoto' s thyroiditis (HT), is a frequent autoimmune disease caused by the autoimmune dysfunction, which is characterized by the infiltration of lymphocytes in the thyroid and the production of thyroid autoantibodies (1). The pathogenesis is complex, involving heredity and environmental factors. And the incidence is higher in women than in men, increasing with age. Genetic factors, radiation exposure, increased iodine intake, infection, smoking, life stress, and the use of amiodarone and interferon can lead to AITD in susceptible individuals, of which $70 \%$ can be attributed to genetic factors. The common model of the onset of AITD is the underlying genetic susceptibility and triggers that trigger and maintain the autoimmune response, which ultimately leads to hypothyroidism or hyperthyroidism. It is well known that thyroid antibody expression positive play an important role in the diagnosis of AITD, and there is growing concern about the high positive rate of thyroid autoantibodies in the population and its adverse effects on human health, including an increased risk of cardiovascular disease and death, an increased rate of abortion among women of childbearing 
age and an impact on the fetal mental development. However, due to its known multiile causes and slow and complicated autoimmune process, we have no good prevention and control measures for AITD.

HT manifests as subacute or destructive thyroiditis and its pathogenesis is thought to be driven by th1 cells. T cells can directly damage the thyroid gland, and the release of thyroid hormones can lead to hyperthyroidism, followed by exposure to thyroid antigens (thyroid peroxidase and thyroglobulin) leading to antibody production and ultimately hypothyroidism. Thyroid gland dysfunction may manifest as clinical thyroid dysfunction ( $0.1-2 \%$ of the population) or subclinical thyroid dysfunction $(10-15 \%$ of the population), depending on the extent of damaged thyroid parenchyma, but HT patients may also have normal thyroid function(3). The major autoantibodies in the peripheral blood of HT patients are thyroid peroxidase antibody (TPOAb) and thyroglobulin antibody (TgAb), but they also occur in about 70\% of GD patients. Thyroid peroxidaseantibody (TPOAb), which indicates thyroid lymphocyte infiltration and follicular epithelial destruction, is detectable in $90 \%$ of HT patients and in $5-24 \%$ of the general population(2)(4). Nearly $50 \%$ of patients diagnosed with HT due to TPOAb positivity have normal thyroid function, while the majority of other patients have subclinical (mild) hypothyroidism, which is characterized by normal FT4 levels and elevated serum TSH, and only a few patients have severe hypothyroidism. It is estimated that about $2.5 \%$ of people with TPOAb-positive can develop noticeable hypothyroidism and about $4.5 \%$ of people with TPOAb-positive and elevated TSH can develop significant hypothyroidism annually(5). TPOAb status (positive or negative) is important in the initial diagnosis of patients, and both the presence and concentration of TPOAb are associated with the beginning of the disease. One study showed that TPOAb increased the adverse risk at $>500 \mathrm{kU} / \mathrm{l}$, while there was no significant difference in the adverse risk at its low levels(6). The exact function of TgAb is unclear, as they do not cause thyroid cell destruction.

In terms of immunology and pathomorphology, the pathogenesis of GD is thought to be related to Thelper type 2(Th2), with T-cell-mediated response and inflammatory infiltration. The major autoantigen in Graves' disease patients is thyroid stimulating hormone receptor (TSHR), and also present in a small number of patients with Hashimoto's thyroiditis(7). Specific thyroid stimulating hormone receptor autoantibody (TRAb) stimulates the formation of TSHR, leading to hyperthyroidism. Generally TRAb refers to any type of antibody that specifically interacts with TSHR. High TSHR antibody levels at diagnosis or positive TSHR antibody at cessation of treatment indicate a high probability of recurrence within 2 years. The stimulating effect of TRAb on thyroid cells may also influence the formation of $\operatorname{TgAb}$ and TPOAb. The level of thyroid autoantibody titer is also important, but the exact mechanism is not clear.

In addition to thyroid autoantibody production and abnormal thyroid hormone production, AITD is histologically involved in targeting $T$ and $B$ lymphocyte infiltration in the thyroid(1). Different immune cell subsets play an important role in the pathogenesis and tissue damage of AITD, among which the activation of regulatory $T$ cells and helper $T$ cell subsets and the imbalance of cytokines secreted by them are important links leading to the pathogenesis of AITD. The main function of regulating $T$ cells is to inhibit the activation and proliferation of CD $4+C D 8+T$ cells and play a negative regulatory role in the 
immune response. The decreased function of regulating $T$ cells can lead to relatively active functions of $\mathrm{CD} 4+\mathrm{CD} 8+\mathrm{T}$ cells and the imbalance of Th1/Th2, resulting in autoimmune disorders dominated by cellular immune hyperfunction(8)(9)(10). Regulatory T cells (Tregs) are primarily responsible for maintaining self-tolerance, and their protective effects have been demonstrated in animal models of several autoimmune diseases. MorrisGP et al. showed that Treg depletion enables thyroiditis induction with mouse thyroglobulin ( $\mathrm{mTg}$ ) in traditionally-resistant mice and mTg-induced, Treg-mediated tolerance protects against EAT(a model for Hashimoto's thyroiditis) induction in genetically-susceptible mice. Meanwhile, they proposed that the phenotype of Treg is CD4 + CD25 + Foxp3 + regulatory T cells. These data demonstrate that tregs are essential in maintaining peripheral tolerance and have potential therapeutic in inducing tolerance(11). In addition, Treg cells have been linked to chronic inflammation and other autoimmune diseases such as systemic lupus erythematosus, Sjogren's syndrome and systemic sclerosis. There are many subsets of regulatory $T$ cells classified from the origin. These subsets have different T cell receptors (TCR), and have different functions in local tissues. FOXP3 + Treg cells are generally regarded as active regulatory $T$ cells. Although many markers have been identified that play an important role in the immunobiology of Tregs, most are not specific to Tregs. In this experiment, CD4 + CD25 + CD127lowT cells were set as Treg cells in the study.

CD25 (also known as IL-2R receptor or a-subunit of IL-2 receptor) is involved in the regulation of T cell function. It is encoded by the CD25 region on chromosome 10p15.1, highly expressed in Tregs, and mediates IL-2 signal transduction, which is essential for the survival and growth of CD25 + CD $4+$ Tregs(12). It is known that FOXP3 is a master regulator for Treg cell differentiation and function. Foxp3 negatively regulates the transcription of IL-2 and up-regulates the expression of IL-2R, making exogenous IL-2 a necessary condition for the survival of Treg cells. It has been shown that the signal transduction of IL-2 is particularly critical to the maintenance of Treg homeostasis and FOXP3 expression. PangdiYang et al. 's study showed that tregs can absorb IL-2 effectively due to the high expression of CD25, thus depleting IL-2 in the environment, leading to the release of granzyme and perforin, and inducing cell apoptosis. CD25 is highly expressed on the surface of treg cells and binds to IL-2 with high affinity, then JAK/STAT signaling pathway is activated, thus maintaining the expression of FOXP3 and the development, proliferation and inhibitory functions of Tregs. Similar to FOXP3 impaired mice, IL2Radeficient mice showed similar lethal lymphoproliferative disease with severe autoimmunity(13). Therefore, it is hypothesized that some genetic variants in the CD25 gene predispose to autoimmune disease by impairs Treg cell function and peripheral tolerance.In fact,a case-control study from Britain reported a significant correlation between CD25 and GD(14). NTregs can inhibit multi-organ autoimmunity induced by CD4 + CD25-T cells in nude mice, and Tregs consumption in vivo increased the incidence of autoimmune diseases including thyroiditis in other resistant mouse.

Interleukin-7 (IL-7) is a crucial cytokine involved in T-cell survival and development, and CD127 expression ensures an appropriate response to IL-7 and maintains T-cell homeostasis(15)(16)(17). IT is known that FOXP3 is a key regulator of Treg cell differentiation and function, and regulatory CD $4+F O X P 3+T$ cells express very little CD127 on their surface,so a combination of CD4, CD25 and CD127 antibody can be used to evaluate the phenotype of Treg cells (CD4 + CD25 + CD127-T cells). CD 4 + CD25 + CD127 (low) T 
cells express high levels of Foxp3 and exert high immunosuppression against effector T cells(18)(19). Studies have shown that the expression of CD127 is decreased on CD4 + T cells and the proportion of CD4 + CD25 + CD127 (low) T cells is increased in the peripheral blood of children with type 1 diabetes with a longer duration, and there is a good correlation between them(20). Currently, CD4, CD25 and CD127 combinations have been used to select $T$ cells for functional research as well as for expansion and adoptive immunotherapy after expansion.

There are two independent signals in the classical T cell activation pathway, One is the specific signal provided by the interaction between the MHC-antigen (Ag) complex on antigen presenting cells (APCs) and $T$ cell receptors (TCRs) on T cells, and the other is the non-antigen-specific signal transmitted by the co-stimulation molecules expressed on the surface of T cells and APCs cells. The costimulatory signaling pathway not only provides a critical positive second signal to initiate, enhance, and maintain the T-cell response, but also provides a critical negative second signal to limit, terminate, or impair the T-cell response. Programmed cell death-1 (PD-1)/programmed cell death ligand (PD-L1) costimulatory pathway is a negative costimulatory pathway discovered in recent years, PD-1 and its ligands PD-L1 (B7-H1, CD274) and PD-L2 (B7-DC, CD273) plays an important role in the induction and maintenance of peripheral tolerance and for the maintenance of the stability and the integrity of $T$ cells. However, the PD1:PD-L1/L2 pathway also mediates potent inhibitory signals to hinder the proliferation and function of T effector cells. Studies have shown that enhancing or blocking this pathway is associated with the immune process of AITD. PD-1, a type I transmembrane glycoprotein, is a co-inhibitory receptor of the immunoglobulin gene superfamily. In humans and mice,the gene encoding PD-1 is composed of 5 exons. Exon 1 encodes signal sequence, exon 2 encodes IgV-like domain, exon 3 encodes stem and TM domain, and exon 4 and 5 encodes cytoplasmic domain.It is mainly expressed in activated T cells and B cells(21). PD-1 is mainly developed in the thymus, and can also be induced to express in various hematopoietic cells by antigen receptor signals and cytokines in the periphery. As mentioned above,PD-1 has two ligands, PD-L1(B7-H1; CD274) and PD-L2(B7-DC; CD273), the difference between PD-L1 and PD-L2 lies in their different affinity for PD-1. The affinity of PD-L2 to PD-1 is three times higher than that of PD-L1, but the expression of PD-L1 is more extensive than that of PD-L2. PD-L1 can be induced to express in various hematopoietic and non-hematopoietic cells as well as various malignant cell, such as B cells, dendritic cells, macrophages, (BM-derived) mast cells and T cells, and can be upregulated further after activation. PD-L2 can be induced to express in dendritic cells, macrophages, peritoneal B cells, memory B cells and (BM-derived) mast cells. After pd-1 binds to its ligand, Intracellular tyrosine residues are phosphorylated and phosphatases are subsequently activated, then proximal TCR signal transduction is inhibited, ultimately inhibiting $T$ cell activation, proliferation, and cytokine secretion(22)(23). Therefore, the interaction between Treg cells expressing PD-1 and PD-L1 and conventional T cells can lead to negative regulation of Treg cells and conventional T cells.

\section{Materials And Methods 1.Research object}


inclusion criteria: the study was conducted between May 2018 and December 2018 and comprised 20 patients with GD [including 4 males and 16 females (median age: $41.00 \pm 17.36$ years, range: $10-69$ years)] and 21 patients with $\mathrm{HT}$ [including 2 males and 19 females (median age: $39.38 \pm 11.87$ years, range: 23-69 years)] sequentially recruited from the endocrinology outpatient clinic of the second affiliated hospital of Dalian Medical University. All patients were newly diagnosed. The qualifying criteria for patients with AITD were based on the Chinese Guidelines for diagnosis and Treatment of Thyroid Diseases. GD was diagnosed in patients with hypermetabolic symptoms, diffuse thyroid enlargement was confirmed by palpation and ultrasoundand (a few cases may have no goiter), reduced TSH level and elevated FT3 and(or) FT4 level,and positive TRAb, elevated thyroid iodine-131 uptake rate and with or without exopthalmos or pretibialmyxedema. HT was diagnosed in patients with diffuse symmetrical goiter with tough, uneven surface or nodules, positive TgAb and(or) TPOAb and normal or elevated TSH.Serum levels of TSH (reference values $0.51-4.94 \mathrm{mlU} / \mathrm{ml})$, free T4 (12-22pmol/L), free T3 (2.77$6.31 \mathrm{pmol} / \mathrm{L})$, TPOAb positive $(>60 \mathrm{IU} / \mathrm{ml})$ and TGAb positive $(>60 \mathrm{IU} / \mathrm{ml})$ were determined by chemiluminescence immunoassay(Centaur XP).

Exclusion criteria included people who also suffer from malignant tumor, immune deficiency or other autoimmune diseases, such as diabetes, systemic lupus erythematosus, inflammatory bowel disease, etc, patients with acute or chronic infectious diseases, such as acute or chronic hepatitis, pneumonia, etc,people who use nonsteroidal drugs or corticosteroids, and pregnant women.

In the same period, twenty healthy persons (mean age 39.45 \pm 13.34 years,range: $22-64$ years) served as a control group (CG). All controls had normal thyroid function, negative thyroid antibody, and no history of related immune disease or recent infection.

\section{Materials}

\subsection{Main Instruments and Equipments}

The experimental materials included Biological purification table(Haier HCB1300V), Inverted microscope( Leica DMI1),Ultrapure water machine (Milli-Q Advantage A10), Centrifuger(Leica DMI1),Single channel pipette(Eppendorf), $4^{\circ} \mathrm{Crefrigerator(Haier),Flow} \mathrm{cytometer(FACS} \mathrm{Calibur} \mathrm{TM} \mathrm{of} \mathrm{Becton,Dickinson}$ ,USA),Disposable medical blood collection device(Becton,Dickinson,USA),Heparin sodium anticoagulant vacuum tube(4ml)(Becton,Dickinson,USA), Centrifuge tube,Latex gloves,etc.

\subsection{Main reagent}

Human lymphocyte separation fluid(TBD company),PBS powder(Wuhan Boster Biological Technology,China),APC Mouse IgG1,APC anti-human CD279(PD-1)Antibody,PE/Cy7 Mouse lgG2b,PE/Cy7 anti-human CD274(B7-H1,PD-L1)Antibody,PE Mouse IgG1,PE anti-human CD25 Antibody,PerCP/Cy5.5 Mouse IgG1, PerCP/Cy5.5 anti-human CD127(IL-7Ra)Antibody, FITC Mouse IgG1, FITC anti-human CD4 Antibody(Biolegend, USA). 


\section{3 reagent preparation}

Blending PBS powder and $1000 \mathrm{ml}$ hydrogen peroxide together in a flask evenly and adjust PH to 7.4 , then the product is autoclaved and sterilized $\left(121^{\circ} \mathrm{C} \otimes 20 \mathrm{~min}\right)$. After cooling, it is stored in the refrigerator at $4^{\circ} \mathrm{C}$ for later use.

\section{Experimental methods}

FCM was used to detect the percentage of CD4 + CD127 + T lymphocytes, CD4 + CD25 + CD127(low)Treg cells and PD1/PDL-1 on Treg cells in peripheral blood.

\section{Experimental steps}

All subjects were in a quiet condition, 2-3ml peripheral blood was extracted from the median cubital vein of the study subjects under aseptic conditions and loaded into an anticoagulant vacuum tube containing $0.2 \mathrm{ml}$ heparin sodium. PBMCs were isolated by Ficoll density-gradient centrifugation and were stained with the following anti-bodies: FITC anti-human CD4,PerCP/Cy5.5 anti-human CD127,PE anti-human CD25,APC anti-human PD-1,PE/Cy7 anti-human PD-L1.Data were acquired in FACS Calibur TM flow cytometer (Becton-Dickinson).

\section{Statistical analysis}

Data analyses were performed using SPSS software, and all data are expressed as mean and SD. Differences were considered significant at $p<0.05$.

\section{Results}

\section{Basic clinical data of enrolled patients and healthy control group}

$\mathrm{GD}(\mathrm{N}=20) \mathrm{HT}(\mathrm{N}=21)$ Healthy control $(\mathrm{N}=20)$

\begin{tabular}{|llll|}
\hline Age(years) & $\mathbf{4 1 . 0 0 \pm 1 7 . 3 6}$ & $\mathbf{3 9 . 3 8} \pm \mathbf{1 1 . 8 7}$ & $\mathbf{3 9 . 4 5} \pm \mathbf{1 3 . 3 4}$ \\
\hline Gender(Male/female) & $4 / 16$ & $2 / 19$ & $1 / 19$ \\
\hline FT3(pmol/l) & $12.90 \pm 9.69$ & $4.48 \pm 0.46$ & $4.66 \pm 0.43$ \\
\hline FT4(pmol/l) & $35.48 \pm 24.40$ & $15.27 \pm 3.23$ & $16.00 \pm 1.80$ \\
\hline TSH(pmol/l) & $0.32 \pm 0.67$ & $6.48 \pm 12.96$ & $1.81 \pm 0.92$ \\
\hline
\end{tabular}

\section{Characteristics of CD4 + CD127 + cells in peripheral blood of patients with AITD}


Compared with the HCs and GD patients, the percentages of CD4 + CD127 + T cells and the MFI of CD127 in CD $4+C D 127+T$ cells in peripheral blood of HT patients were not significantly different $(p>0.05)$.

Compared with the HCs and HT patients, the percentages of CD4 + CD127 + T cells and the MFI of CD127 in CD $4+$ CD127 + T cells in peripheral blood of GD patients were not significantly different $(p>0.05)$.

\begin{tabular}{|llll|}
\hline & GD $(\mathbf{N}=\mathbf{2 0})$ & $\mathrm{HT}(\mathbf{N}=\mathbf{2 1})$ & Healthy control $(\mathbf{N}=\mathbf{2 0})$ \\
\hline $\mathrm{CD} 4+\mathrm{CD} 127+\mathrm{T}$ cell & $34.49 \pm 8.19$ & $30.07 \pm 8.96$ & $26.26 \pm 9.12$ \\
\hline $\mathrm{MFI}$ & $1801.5 \pm 944.3$ & $2212.1 \pm 977.9$ & $1922.0 \pm 901.9$ \\
\hline
\end{tabular}

\section{Characteristics of Treg (CD4 + CD25 + CD127low) cells in peripheral blood of AITD patients}

Compared with the HCs and GD patients, the percentage of CD4 + T cells in peripheral blood of HT patients were not significantly different $(p>0.05)$, but the percentage of CD $4+C D 25+C D 127$ lowT cells in CD4 $+T$ cells was $(2.94 \pm 0.61)$ in HT patients vs $(5.60 \pm 1.26)$ in control group, the difference was statistically significant $(P<0.05)$. The results showed that the proportion of CD4 + CD25+CD127lowT cells in peripheral blood of $\mathrm{HT}$ patients was lower than that of healthy control group.Compared with the HCs and HT patients, the percentage of CD4 + T cells in peripheral blood of GD patients were not significantly different $(p>0.05)$, the percentage of CD $4+C D 25+C D 127$ lowT cells in CD $4+T$ cells was $(5.78 \pm 1.74)$ in GD patients vs $(2.94 \pm 0.61)$ in $\mathrm{HT}$ patients, the difference was statistically significant $(\mathrm{P}<$ $0.05)$, but there was no statistical difference $(P>0.05)$ as compared to control group.

\section{Characteristics of PD-1 expression on Tregs in peripheral blood of AITD patients}

Compared with the HCs and GD patients, the ratio of PD-1 + Treg cells in treg cells in peripheral blood was $(6.54 \pm 1.80)$ in $\mathrm{HT}$ patients vs $(1.22 \pm 0.70)$ in healthy control, $(6.54 \pm 1.80)$ in HT patients vs $(3.87 \pm 3.43)$ in GD patients,there was statistical difference $(P<0.05)$. The results showed that the ratio of PD- $1+$ Treg cells in treg cells in peripheral blood of HT patients was higher than that of the HCs and GD patients.Compared with the HCs and HT patients, the ratio of PD-1 + Treg cells in treg cells in peripheral blood was $(3.87 \pm 3.43)$ in GD patients vs $(1.22 \pm 0.70)$ in healthy control, $(3.87 \pm 3.43)$ in GD patients vs $(6.54 \pm 1.80)$ in $\mathrm{HT}$ patients,there was statistical difference $(P<0.05)$. The results showed that the ratio of PD-1 + Treg cells in treg cells in peripheral blood of GD patients was higher than that of the HCs and lower than that of HT patients.

\section{Characteristics of PD-L1 expression on Tregs in peripheral blood of AITD patients}

Compared with the HCs and GD patients, the ratio of PD-L1 + Treg cells in treg cells in peripheral blood was $(4.56 \pm 1.66)$ in $\mathrm{HT}$ patients vs $(0.97 \pm 0.37)$ in healthy control, $(4.56 \pm 1.66)$ in HT patients vs $(2.01 \pm$ $1.66)$ in $G D$ patients,there was statistical difference $(P<0.05)$. The results showed that the ratio of PD-L1 
+ Treg cells in treg cells in peripheral blood of HT patients was higher than that of the HCs and GD patients. Compared with the HCs and HT patients, the ratio of PD-L1 + Treg cells in treg cells in peripheral blood was $(2.01 \pm 1.66)$ in GD patients vs $(0.97 \pm 0.37)$ in healthy control, $(2.01 \pm 1.66)$ in GD patients vs $(4.56 \pm 1.66)$ in $\mathrm{HT}$ patients, there was statistical difference $(P<0.05)$. The results showed that the ratio of PD-L1 + Treg cells in treg cells in peripheral blood of GD patients was higher than that of the HCs and lower than that of HT patients.

\begin{tabular}{|llll|}
\hline & $\mathrm{GD}(\mathrm{N}=20)$ & $\mathrm{HT}(\mathrm{N}=21)$ & Healthy control $(\mathrm{N}=20)$ \\
\hline Treg(CD4+CD25 + CD127low $)$ & $5.78 \pm 1.74$ & $2.94 \pm 0.61$ & $5.60 \pm 1.26$ \\
\hline PD-1 + Treg & $3.87 \pm 3.43$ & $6.54 \pm 1.80$ & $1.22 \pm 0.70$ \\
\hline PD-L1 + Treg & $2.01 \pm 1.66$ & $4.56 \pm 1.66$ & $0.97 \pm 0.37$ \\
\hline
\end{tabular}

\section{Discussion}

Thyroid disease is a common disease in the endocrine system, mainly including thyroid dysfunction and autoimmune thyroid disease (AITD). AITD, a most common cause of thyroid dysfunction, has significantly increased in prevalence and incidence over the past decades. AITD mainly includes Hashimoto's thyroiditis (HT) and Graves' disease (GD). HT and GD are characterized by the breakdown of immune tolerance and abnormal expression of key cytokines. However, the pathogenesis of AITD is still unclear. Currently, it is generally accepted that AITD result from a dysregulation of the immune system leading to an immune attack on the thyroid and the common pathological feature of AITD is the presence of lymphocyte infiltrates within the thyroid. In terms of precision medicine, our understanding of the disease has been gradually transformed from the macroscopic clinical disease phenotype into the microscopic gene. In the future, the diagnosis and treatment of the disease will be intervened in the susceptibility and treatment at the genetic level. Little is known about the correlation between Treg cells and the damage factors associated with AITD at this stage.

The downregulation mechanism of CD127 has drawn attention, in some animal experiments and in vitro models, it was mainly associated with $T$ cell activation(24). The downregulation of CD127 among CD4 + $T$ cell pool (not only infected CD $4+T$ cells) reflects that chronic viral infection lead to immune activation. Decreased CD127 expression in HIV-infected patients is closely related to the rate of disease progression, the loss of CD $4+T$ cell and the impairment of protective immune function(25). The reduction of CD127 significantly were also found in diseases characterized by $T$ cell activation, namely non-infectious chronic inflammation such as chronic allergies and asthma(26).In addition, studies have shown the expression of CD127 is altered in patients with rheumatoid arthritis(27). Assessing CD127 levels on CD4 + T cells may have important therapeutic implications, and two separate studies of non-obese diabetic mice have shown that blocking of CD127 can induce complete remission and recovery of autoimmune diabetes by changing the function of Treg cells (28)(29). In this study, there were no statistically significant differences in the ratio of CD4 + CD127 + T cells and the mean fluorescence intensity (MFI) of CD127 in 
the peripheral blood of HCs, HT patients and GD patients, suggesting that $\mathrm{t}$ cell immune abnormality caused by altered expression of CD127 was not obvious in AITD. Considering that the downregulation of CD127 by t cells activation may be related to the course of disease,and activated $T$ cells accumulate continuously during the development of AITD. In this study, no obvious change in the ratio of CD $4+$ CD127 + T cells and the MFI of CD127 because the subjects were newly diagnosed with AITD.

Th1-driven autoimmune response has long been considered a major factor in the development of HT, but recent studies have shown that Treg cells play a part in the pathogenesis of AITD especially HT by inhibiting peripheral reactive $T$ cells and thereby inhibiting the autoimmune response, and play an important regulatory role in the development of other lymphocytes. Treg cell has been found in lymphoid organs and even in non-lymphoid tissues, suggesting that it plays a role in preventing immune damage and can also play non-immune roles, such as maintaining homeostasis and tissue repair. Immune-related functions of Treg cells in non-lymphocyte tissues include inhibition of T cell response, and Treg cells are recruited to specific sites by specific receptors to modulate immune response to infectious pathogens and tolerance to symbiotic organisms.More and more evidence shows that Treg cells are increased in damaged tissues and play an important role in tissue repair, not only by regulating immunity, but also by affecting the regeneration of original non-immune cells in tissues. In damaged skeletal muscle in mice, a subset of Treg cell was found to promote muscle repair by expressing amphiregulin(a type of epidermal growth factor). However, we also found that Treg cells are unstable in that they can lose Foxp3 in an inflammatory response and manifest complex potential pathogenicity. To some extent the instability is due to reduced IL-2 signaling or the inactivation of certain cytokines. In clinical work, we should strive to use Treg cells to modulate immunity and promote the repair, and eliminate the adverse effects of Treg cells, which requires us to have a deeper understanding of Treg cells. Conventional Tregs are characterized by the expression of forkhead box P3 (Foxp3). It has been revealed in humans that Foxp3 + is involved in multiple inhibitory mechanisms to regulate T cells. Foxp3 mutation leads to functional deficiency of Tregs and causes immunomodulatory diseases, manifested as multi-organ autoimmunity, including diabetes mellitus, thyroiditis, etc. The continuous expression of Foxp3 maintains the stability of Tregs. But due to Foxp3 is expressed in the nucleus, we need to destroy the cell membrane during detection and other procedures, which makes it limited in clinical application. It is thought that although Foxp3 is a characteristic surface factor for tregs, it is not a major regulator of Tregs development. Many studies have found that the expression of CD127 in Tregs is lower than that in conventional t cells, and CD127 expression is negatively correlated with Foxp3, which was related to the interaction between Foxp3 and CD127 promoter. Consistent with the research conclusion of Gerald P. Morris et al, we assessed by flow cytometry that the number of Treg cells in peripheral blood of HT patients was significantly lower than that of healthy control group, which proved that Treg cells (CD4 + CD25+ CD127low) played an important role in HT. There was no significant difference in the number of Treg cells in peripheral blood of GD patients and healthy control group, demonstrating that the role of Treg cells in Graves' disease is unclear.

Tregs can express PD-1 and ligand PD-L1 simultaneously, which deserves attention. The respective expression of PD-L1 and PD-1 on Tregs formed a complex immune contact, which can regulate immune 
stability and inhibit t cell activation by providing a novel inhibiting mechanism. Antibody-mediated blocking of the PD-1/PD-L1 interaction has been applied in a variety of clinical cancer models and cancer patients to enhance anti-tumor immunity and reduce tumor response. Recent data show that blocking PD1/PD-L1 signaling pathway can reduce the inhibitory effect of PD-1 pathway on T cells, promote the antitumor activity of activated T cells, and prolong the remission period of tumor. However, the complicated relationship between the PD-1/PD-L1 pathway and Tregs has not been fully clarified. Some studies have suggested that the activation of PD-1 on Treg cells inhibited the response of anti-CD3. This pathway is composed of PD-1 and its ligands PD-L1 and PD-L2, can adjust immunity effectively and maintain the balance between the stimulation and suppression signals required for self-tolerance. PD-1/PD-L1 interactions regulate the tolerance of CD4 in peripheral at multiple sites. There are many potential bidirectional interactions between PD-1 and PD-L1. At present, the correlation between the expression level of PD-1 and its functions is not clear.

It has been found that PD-L1 can promote the development and function of iTreg cells by inducing and maintaining Foxp3 expression, and can induce naive CD4 + T cells differentiate into Treg cells by inhibiting Akt/mTOR signal cascade. PD-L1 can control the development of Treg cells in lymphoid organs, which plays an important role in maintaining immune homeostasis, can promote the development of Treg cells in target tissues to prevent immune-mediated tissue damage, and maintain and enhance Tregs function in inflammatory microenvironment to balance pathogenic Teff cells effectively. Although many studies have shown that PD-Ls can inhibit T cell proliferation and cytokine production, it has also been found that PD-Ls can enhance T cell activation. The consequences of these conflicting causes are unclear and remain controversial. Some studies have shown that PD-L1 increases $T$ cell proliferation by inhibiting INF- - -induced nitric oxide. These findings suggest that some positive effects of PD-L1 and PD-L2 can be interpreted as suppression of negative signals. PD-L1 expression is seen on pancreatic beta cells and may function to limit self-reactive $T$ cells at the target tissue. Ansari et al. demonstrated the protective role of PD-L1 interactions in T1D. Estrogen-mediated immunomodulation involves reduced activation of effector T cells, potentiation of Treg cells, and enhanced expression of the PD-1 costimulatory pathway, which may explain the susceptibility of female patients to AITD.

PD-1/PD-L1 immunoregulatory pathways is highly complex. In this experiment, comparing the expression of PD-1/PD-L1 on Treg cells in peripheral blood between AITD patients and healthy controls, it was found that PD-1/PD-L1 + Treg cells in peripheral blood of HT patients and GD patients were higher than those in control group, but the expression of PD-1 and PD-L1 on Treg cells in GD patients was not as high as that of HT patients. The trend of PD-1/PD-L1 is inversely proportional to the number of Tregs, suggesting that PD-1/PD-L1 is related to the number of Tregs. It has been suggested that PD-1 pathway restricts the activity of T cells by blocking Akt/mTOR pathway, which increase PTEN and block the differentiation and maintenance of Foxp3 + Tregs, the PD-1 pathway may negatively regulate its own function with the loss of PTEN and increase of PD-L1 expression. Therefore, increased PTEN expression through PD-1 signaling may lead to decreased PD-L1 expression and be used to down-regulate the development and(or) function of the negative feedback loop of Tregs. This hypothesis allows for a extensive diversity of T cells, ensuring that the appropriate T cell subsets rapidly respond to immune challenges. Our group 
demonstrated that increased expression of Blimp-1 in aborted mice with AIT may inhibit the PD-1/PD-L1 pathway and then inhibit the mTOR signal, further affecting the function and number of Tregs. Tregs induced by the PD-1 pathway can also help maintain immune homeostasisn and prevent autoimmunity, while allowing protective immunity. In this clinical experiment, it was found that PD-1 and PD-L1 on Treg cells in peripheral blood of AITD patients increased. PD-1 and PD-L1 on Treg cells can combine with those on other effector T cells and APC, suggesting that the change of PD-1/PD-L1 expression on Treg cells in AITD can not only regulate the number of treg cells but also Influence the number and function of other effector T cells and APC.

From what has been discussed above, treg cells and PD-1/PD-L1 play a role in AITD, especially in Hashimoto's thyroiditis. The increased expression of PD-1/PD-L1 on Treg cells can transmit inhibitory signals to inhibit the number and function of Treg cells, resulting in the decrease in the number of Treg cells and the decline in the function of effector T cells, leading to the occurrence of autoimmune thyroiditis dominated by cellular Immune hyperfunction. As mentioned above, antigen specific Treg cells can effectively regulate immunity by a variety of cytokines, we can make our desired alloantigen specific tregs for immune regulation or make tregs expressing specific TCR for targeted therapy, which is feasible in modern genetics and genomic technologies. In the next few years, these therapies are likely to be used clinically, just as monoclonal antibodies are now, to treat diseases more effectively and improve the survival of the patients.

\section{limitation of the study}

Firstly, the number of patients enrolled in this study was relatively small, we will continue to collect clinical specimens in the later stage to expand the sample size and enhance the credibility of the study. Secondly, we only studied the changes of CD $4+C D 127+T$ cells, treg cells and their surface factors PD1/PD-L1 in peripheral blood, it could not represent the changes of CD $4+C D 127+T$ cells, Treg cells and their surface factors PD-1/PD-L1 in thyroid tissue. Thirdly, in this study, we only analyzed the ratio of treg cells and PD-1 /PD-L1 in peripheral blood of patients with AITD and their correlation, but the specific mechanism pathway was not further studied. Next, our research group will study related pathways to further clarify the specific changes and mechanism of tregs and PD-1 /PD-L1 in AITD.

\section{Conclusions}

The ratio of treg cells in peripheral blood of HT patients was lower than that of HCs and GD patients, and the ratio of treg cells in peripheral blood of GD patients was not significantly different compared with that of HCs, indicating that treg cells play an obvious role in hashimoto's thyroiditis, but not obvious in Graves' disease. The expressions of PD-1 and PD-L1 on treg cells in peripheral blood of HT patients and GD patients were both increased, but the expression of PD-1 and PD-L1 on treg cells in peripheral blood of GD patients was not as high as that of HT patients, PD-1/PD-L1 is inversely proportional to the number of Tregs, suggesting that PD-1/PD-L1 is associated with changes in the number of Tregs and autoimmune thyroid disease.

Page $12 / 22$ 


\section{Abbreviations}

Autoimmune thyroid disease--AITD; Programmed cell death-1--PD-1; programmed cell death ligand --PD-L1; graves disease --GD; Hashimotos'thyroiditis --HT; healthy controls --HCs; peroxidase antibody --TPOAb; thyroglobulin antibody --TgAb; thyroid stimulating hormone receptor --TSHR; stimulating hormone receptor autoantibody --TRAb; Regulatory T cells --Tregs; T cell receptors -TCR; antigen presenting cells --APCs

\section{Declarations}

Ethics approval and conset to participate

Written informed conset for publication of this paper was obtained from Dalian Medical University and all authors.

Conset for publication

Not applicable.

Availability of data and material

The datasets used and analyzed during the current study are available from the corresponding author on reasonabie request.

Competing interests

The authors declared no potential conflicts of interest with respect to the research, author-ship, and publication of this article.

Funding

The authors declare that there is no funding associated with this manuscript.

Authors' contributions

LC and XG wrote the manuscript. LC drew the tables and created the figure in the manuscript. $\mathrm{HL}$ contributed to the conception and writing. $\mathrm{HL}, \mathrm{HZ}$, and $\mathrm{XG}$ revised the manuscript. All authors read and approved the final manuscript.

Acknowledgements

Not applicable. 


\section{References}

1. Tomer Y. Mechanisms of autoimmune thyroid diseases: from genetics to epigenetics. Annual review of pathology. 2014;9:147 - 56.

2. Hollowell JG, Staehling NW, Flanders WD, Hannon WH, Gunter EW, Spencer CA, et al. Serum TSH, $T(4)$, and thyroid antibodies in the United States population (1988 to 1994): National Health and Nutrition Examination Survey (NHANES III). The Journal of clinical endocrinology and metabolism. 2002;87(2):489 - 99.

3. Stathatos N, Daniels $\mathrm{GH}$. Autoimmune thyroid disease. Current opinion in rheumatology. 2012;24(1):70 - 5.

4. Pearce EN, Farwell AP, Braverman LE. Thyroiditis. The New England journal of medicine. 2003;348(26):2646-55.

5. Vanderpump MP. How should we manage patients with mildly increased serum thyrotrophin

6. concentrations? Clinical endocrinology. 2010;72(4):436 - 40.

7. Greer LG, Casey BM, Halvorson LM, Spong CY, McIntire DD, Cunningham FG. Antithyroid antibodies and parity: further evidence for microchimerism in autoimmune thyroid disease. American journal of obstetrics and gynecology. 2011;205(5):471.e1-4.

8. Effraimidis G, Wiersinga WM. Mechanisms in endocrinology: autoimmune thyroid disease: old and new players. European journal of endocrinology. 2014;170(6):R241-52.

9. Maloy KJ, Salaun L, Cahill R, Dougan G, Saunders NJ, Powrie F. CD4 + CD25 + T(R) cells suppress innate immune pathology through cytokinedependent mechanisms. The Journal of experimental medicine. 2003;197(1):111-9.

10. Vasu C, Dogan RN, Holterman MJ, Prabhakar BS. Selective induction of dendritic cells using granulocyte macrophage-colony stimulating factor, but not fms-like tyrosine kinase receptor 3-ligand, activates thyroglobulin-specific CD4+/CD25 + T cells and suppresses experimental autoimmune thyroiditis. Journal of immunology (Baltimore, Md: 1950). 2003;170(11):5511-22.

11. Lichiardopol C, Mota M. The thyroid and autoimmunity. Romanian journal of internal medicine = Revue roumaine de medecine interne. 2009;47(3):207 - 15.

12. Morris GP, Brown NK, Kong YC. Naturally-existing CD4(+)CD25(+)Foxp3(+) regulatory T cells are required for tolerance to experimental autoimmune thyroiditis induced by either exogenous or endogenous autoantigen. Journal of autoimmunity. 2009;33(1):68-76.

13. Cerosaletti K, Schneider A, Schwedhelm K, Frank I, Tatum M, Wei S, et al. Multiple autoimmuneassociated variants confer decreased IL-2R signaling in CD4 + CD25(hi) T cells of type 1 diabetic and multiple sclerosis patients. PloS one. 2013;8(12):e83811. 
14. Bayer AL, Yu A, Adeegbe D, Malek TR. Essential role for interleukin-2 for CD4(+)CD25(+) T regulatory cell development during the neonatal period. The Journal of experimental medicine. 2005;201(5):769 $-77$.

15. Brand OJ, Lowe CE, Heward JM, Franklyn JA, Cooper JD, Todd JA, et al. Association of the interleukin-2 receptor alpha (IL-2Ralpha)/CD25 gene region with Graves' disease using a multilocus test and tag SNPs. Clinical endocrinology. 2007;66(4):508 - 12.

16. Schluns KS, Kieper WC, Jameson SC, Lefrancois L. Interleukin-7 mediates the homeostasis of naive and memory CD8 T cells in vivo. Nature immunology. 2000;1(5):426 - 32.

17. Chetoui N, Boisvert M, Gendron S, Aoudjit F. Interleukin-7 promotes the survival of human CD4 + effector/memory $T$ cells by up-regulating Bcl-2 proteins and activating the JAK/STAT signalling pathway. Immunology. 2010;130(3):418 - 26.

18. Fry TJ, Mackall CL. The many faces of IL-7: from lymphopoiesis to peripheral T cell maintenance. Journal of immunology (Baltimore, Md: 1950). 2005;174(11):6571-6.

19. Hartigan-O'Connor DJ, Poon C, Sinclair E, McCune JM. Human CD4 + regulatory T cells express lower levels of the IL-7 receptor alpha chain (CD127), allowing consistent identification and sorting of live cells. Journal of immunological methods. 2007;319(1-2):41-52.

20. Sakaguchi S, Miyara M, Costantino CM, Hafler DA. FOXP3 + regulatory T cells in the human immune system. Nature reviews Immunology. 2010;10(7):490-500.

21. Moniuszko M, Glowinska-Olszewska B, Rusak M, Jeznach M, Grubczak K, Lipinska D, et al. Decreased CD127 expression on CD4 + T-cells and elevated frequencies of CD4 + CD25 + CD127-Tcells in children with long-lasting type 1 diabetes. Clinical \& developmental immunology. 2013;2013:459210.

22. Ishida $Y$, Agata $Y$, Shibahara $K$, Honjo T. Induced expression of PD-1, a novel member of the immunoglobulin gene superfamily, upon programmed cell death. The EMBO journal. 1992;11(11):3887-95.

23. Okazaki T, Honjo T. The PD-1-PD-L pathway in immunological tolerance. Trends in immunology. 2006;27(4):195-201.

24. Riley JL. PD-1 signaling in primary T cells. Immunological reviews. 2009;229(1):114 - 25.

25. Crawley AM, Angel JB. The influence of HIV on CD127 expression and its potential implications for IL-7 therapy. Seminars in immunology. 2012;24(3):231 - 40.

26. Kiazyk SA, Fowke KR. Loss of CD127 expression links immune activation and CD4(+) T cell loss in HIV infection. Trends in microbiology. 2008;16(12):567 - 73.

27. Moniuszko M, Kowal K, Jeznach M, Rusak M, Dabrowska M, Bodzenta-Lukaszyk A. Phenotypic correlations between monocytes and CD4 + T cells in allergic patients. International archives of allergy and immunology. 2013;161(2):131 - 41.

28. Holmen N, Lundgren A, Lundin S, Bergin AM, Rudin A, Sjovall H, et al. Functional CD4 + CD25high regulatory $T$ cells are enriched in the colonic mucosa of patients with active ulcerative colitis and increase with disease activity. Inflammatory bowel diseases. 2006;12(6):447 - 56. 
29. Penaranda C, Kuswanto W, Hofmann J, Kenefeck R, Narendran P, Walker LS, et al. IL-7 receptor blockade reverses autoimmune diabetes by promoting inhibition of effector/memory $\mathrm{T}$ cells.

Proceedings of the National Academy of Sciences of the United States of America.

2012;109(31):12668-73.

30. Lee LF, Logronio K, Tu GH, Zhai W, Ni I, Mei L, et al. Anti-IL-7 receptor-alpha reverses established type 1 diabetes in nonobese diabetic mice by modulating effector T-cell function. Proceedings of the National Academy of Sciences of the United States of America. 2012;109(31):12674-9.

\section{Figures}

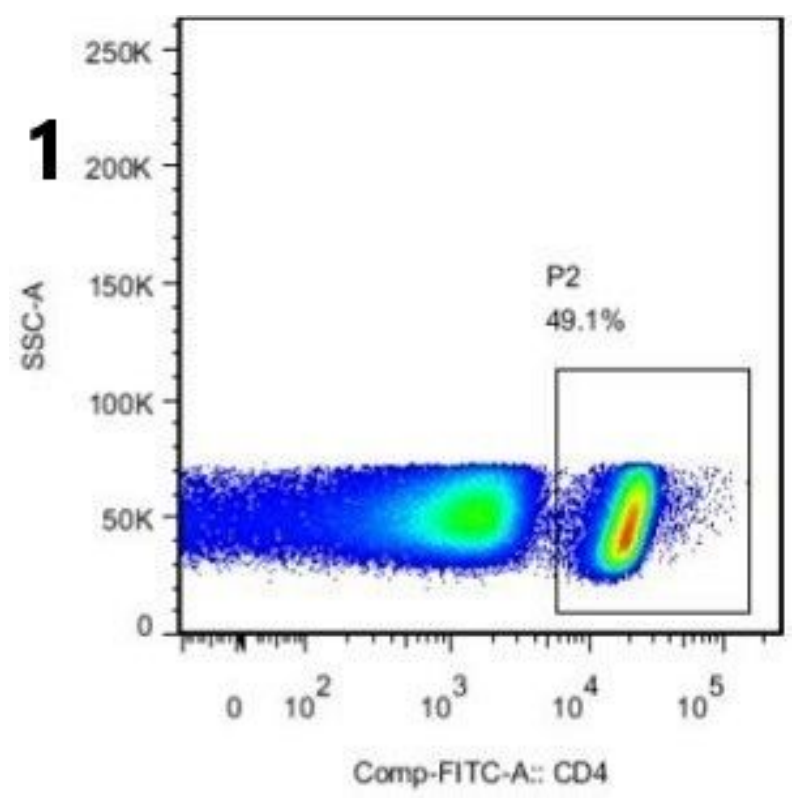

Figure 1

The ratio of CD4+T cells to total lymphocyte (healthy control group) 


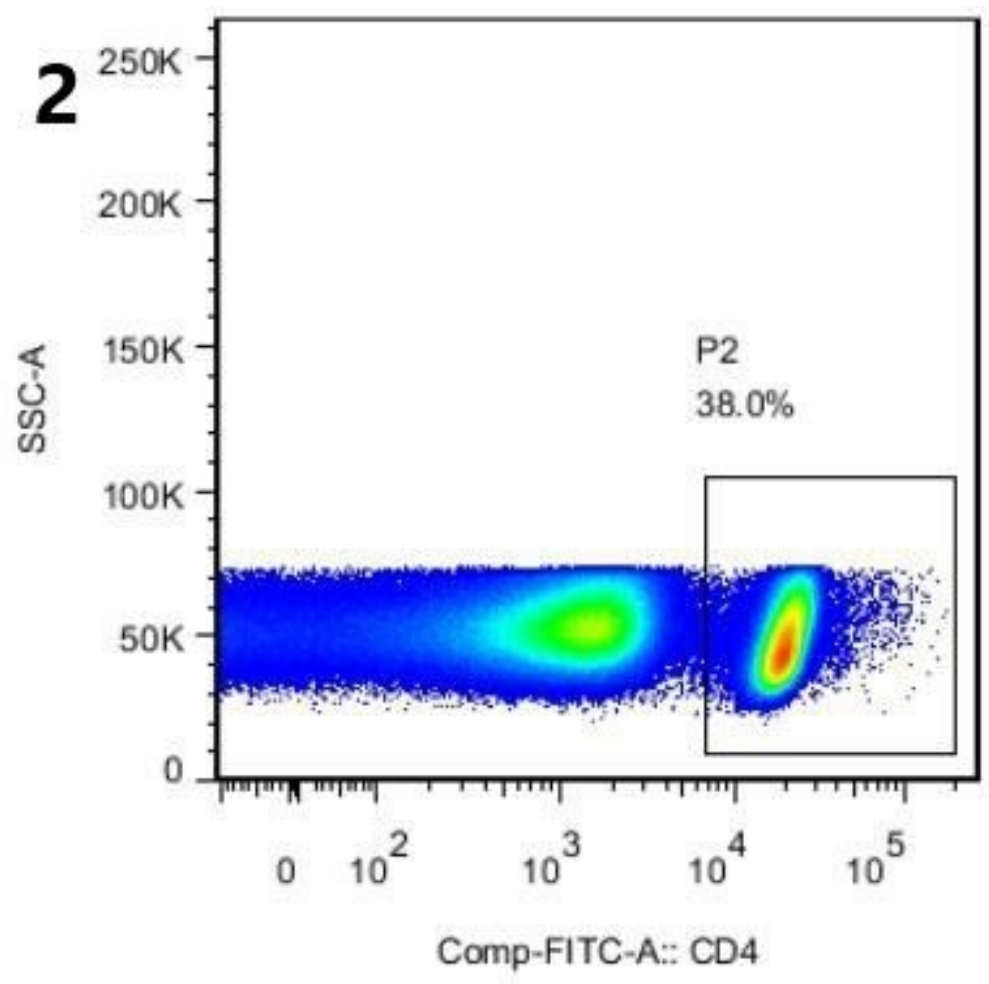

Figure 2

The ratio of CD4+T cells to total lymphocyte (HT patients)

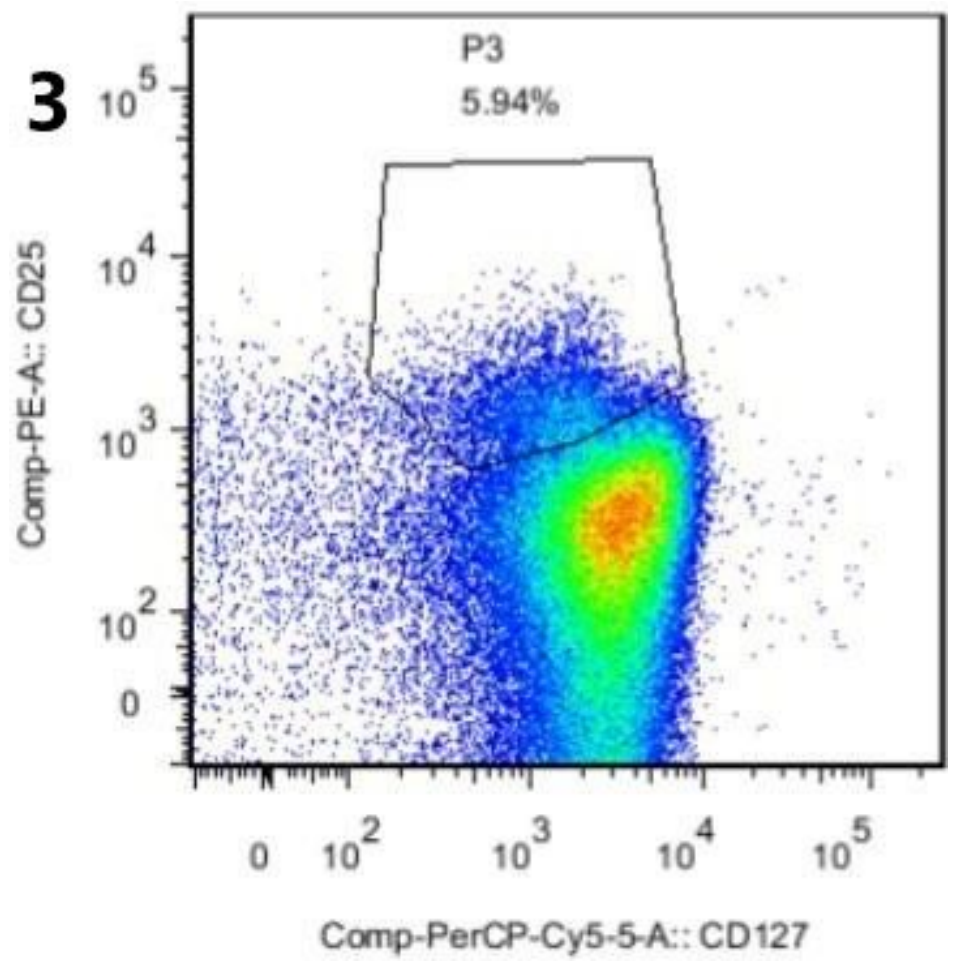

Figure 3

The ratio of $C D 25+C D 127$ lowT cells to $C D 4+T$ cells (healthy control group) 


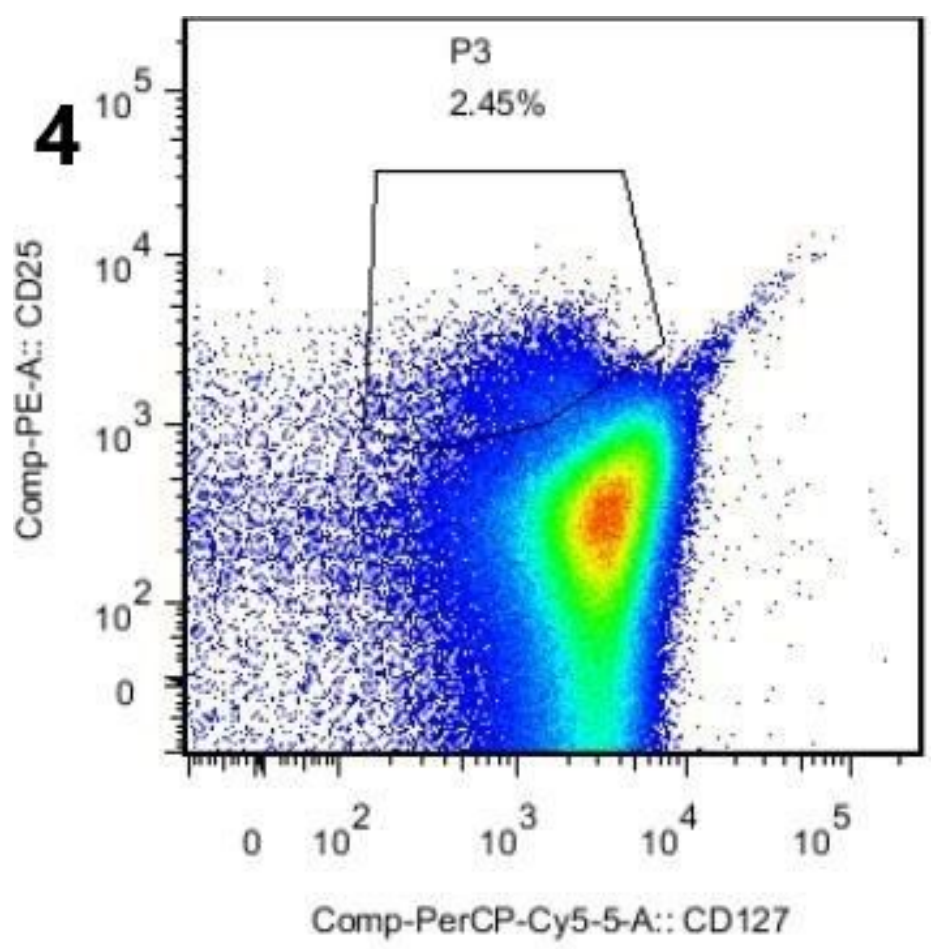

Figure 4

The ratio of CD25+CD127lowT cells to CD4+T cells (HT patients)

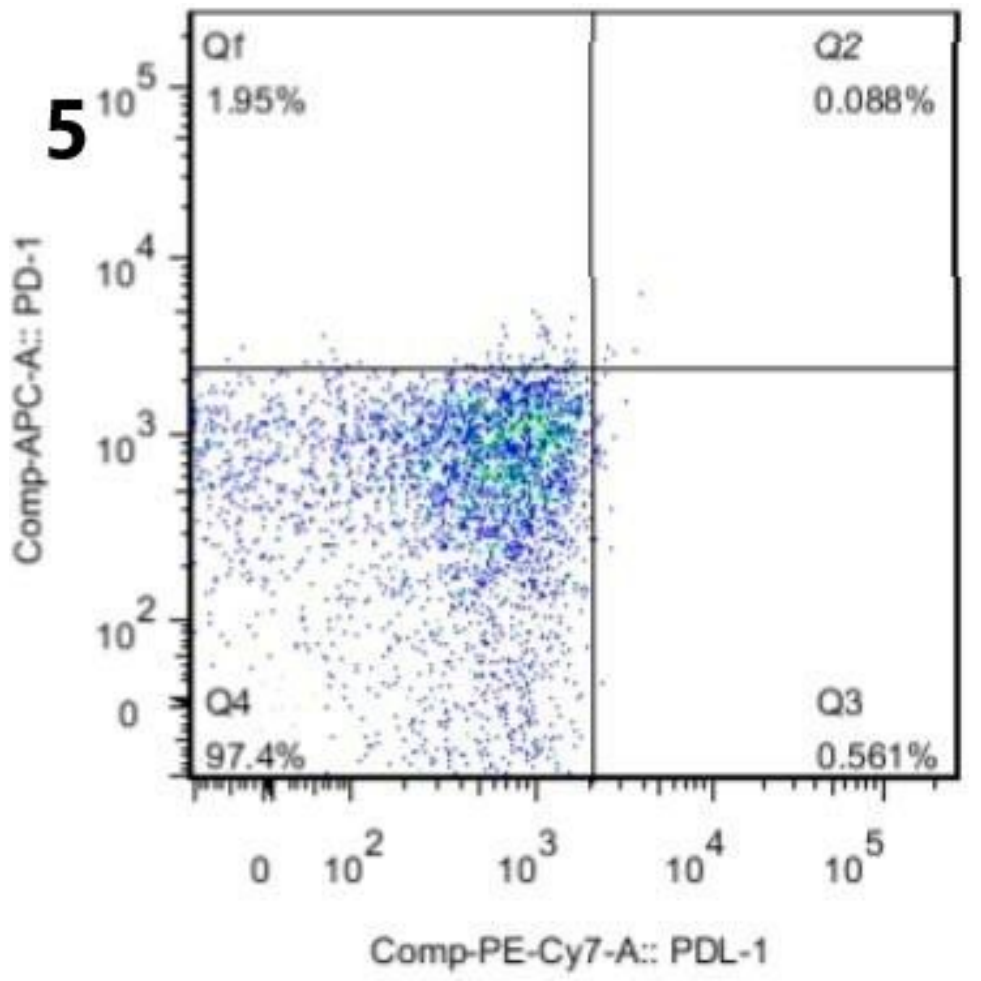

Figure 5

The ratio of PD-1+Treg cells, PD-L1+Treg cells to CD4+CD25+CD127lowT cells (healthy control group) 


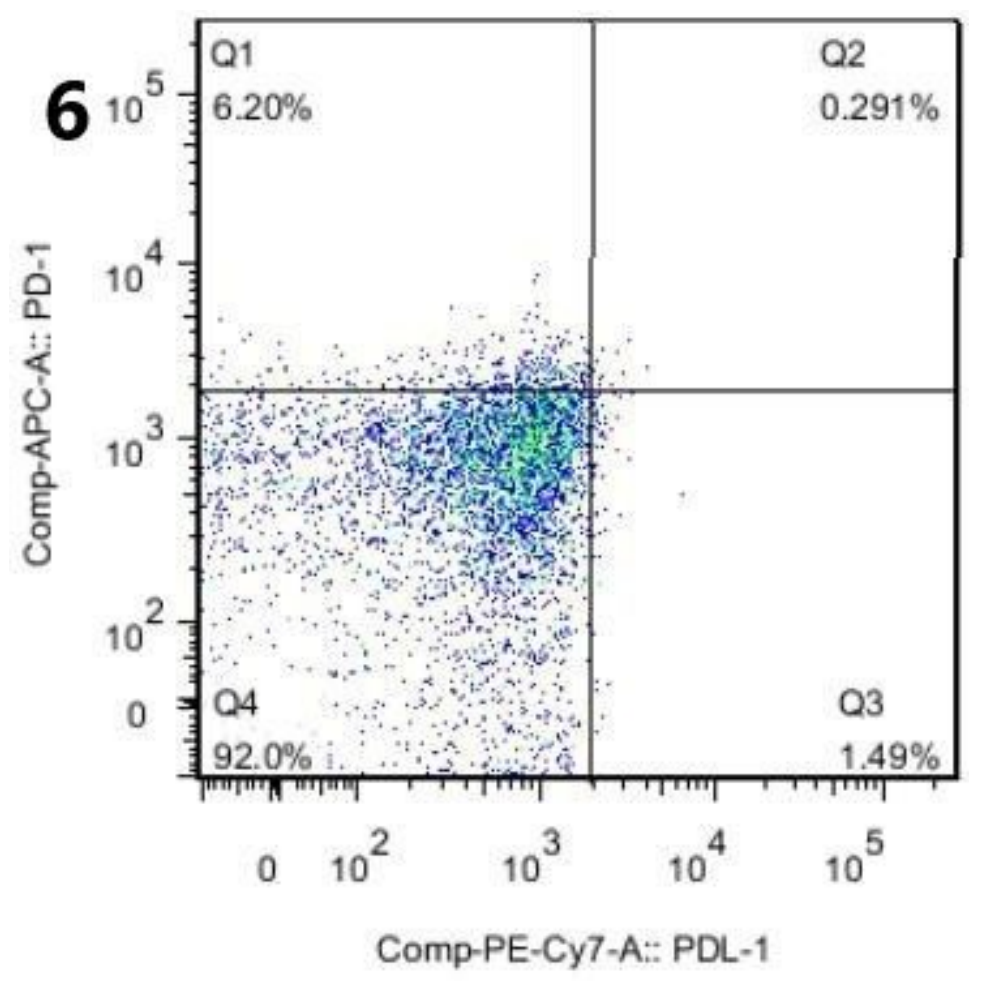

Figure 6

The ratio of PD-1+Treg cells, PD-L1+Treg cells to CD4+CD25+CD127lowT cells (HT patients)

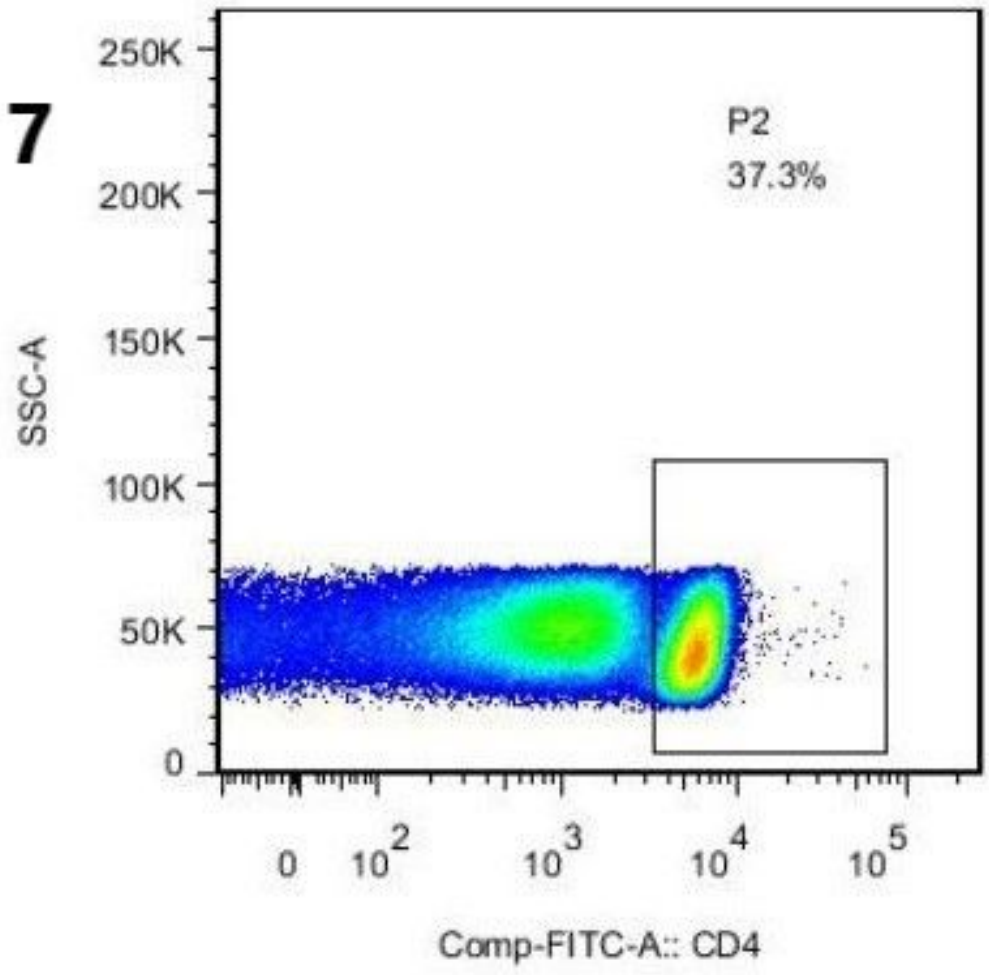

Figure 7

The ratio of CD4+T cells to total lymphocyte (healthy control group) 


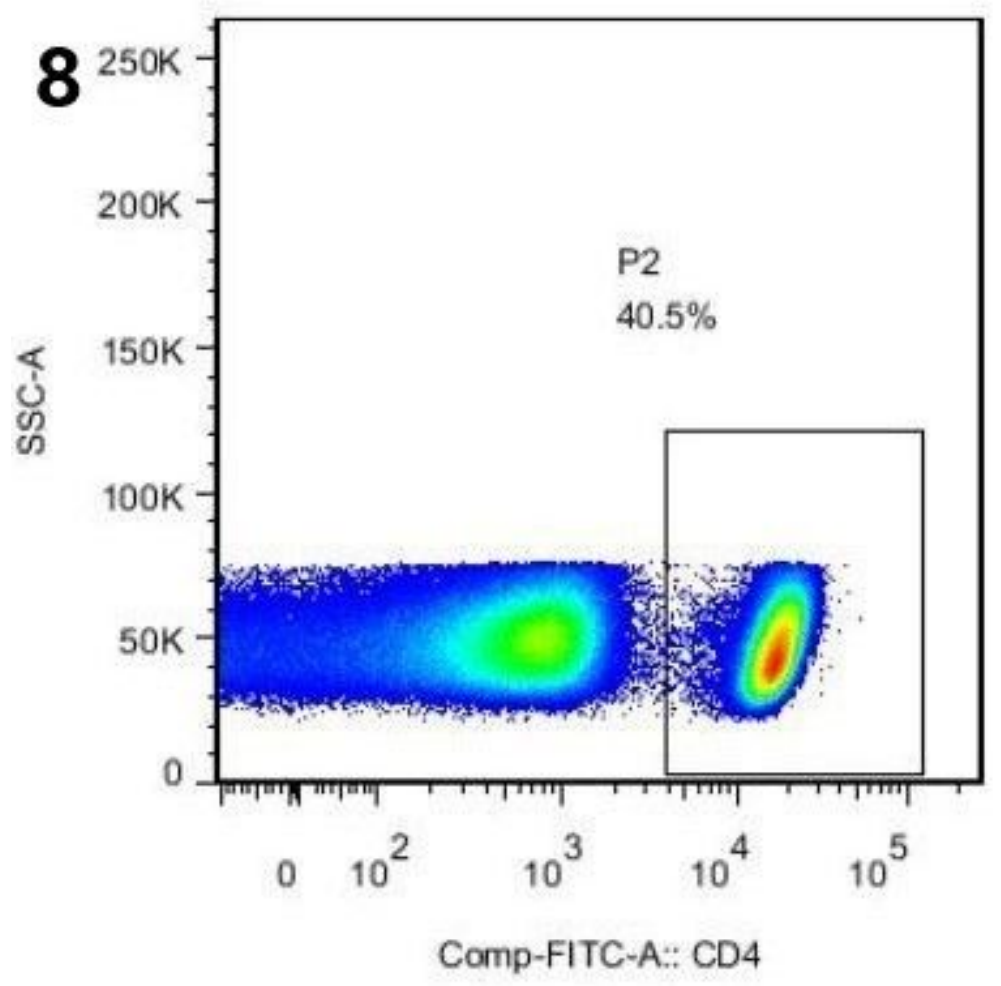

Figure 8

The ratio of CD4+T cells to total lymphocyte (GD patients)

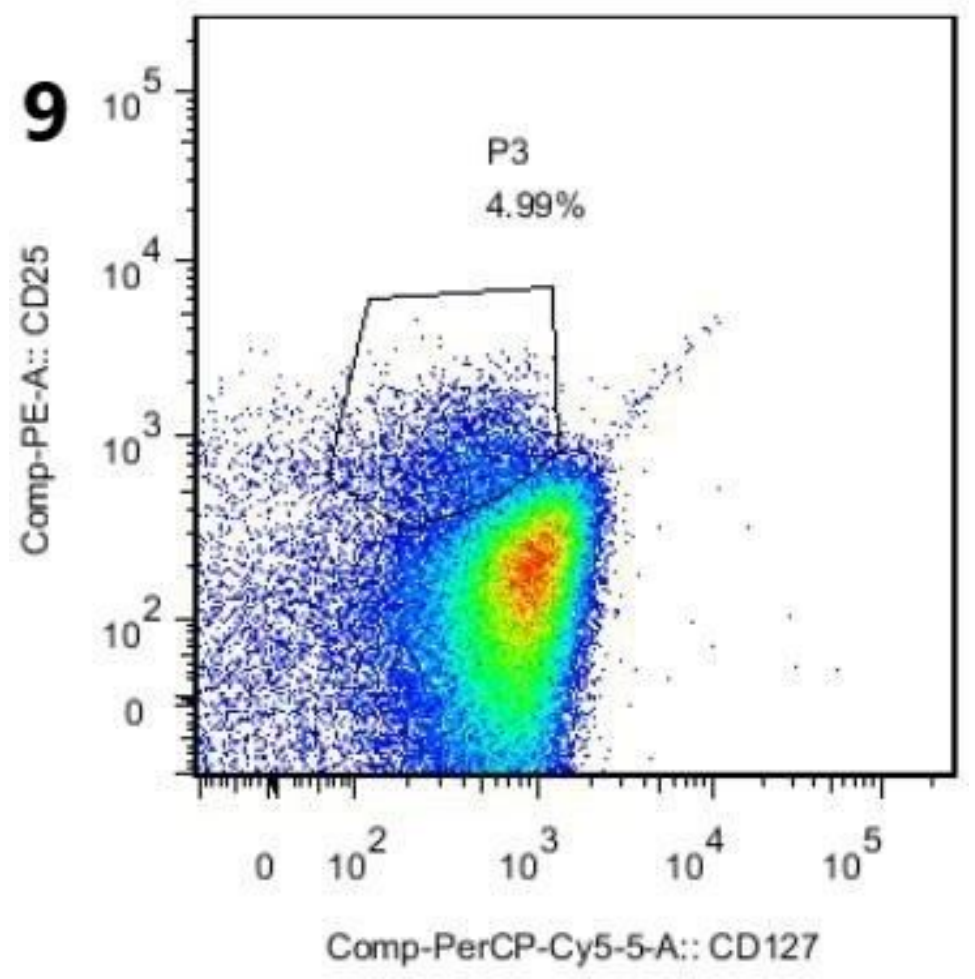

Figure 9

The ratio of $C D 25+C D 127$ lowT cells to $C D 4+T$ cells (healthy control group) 


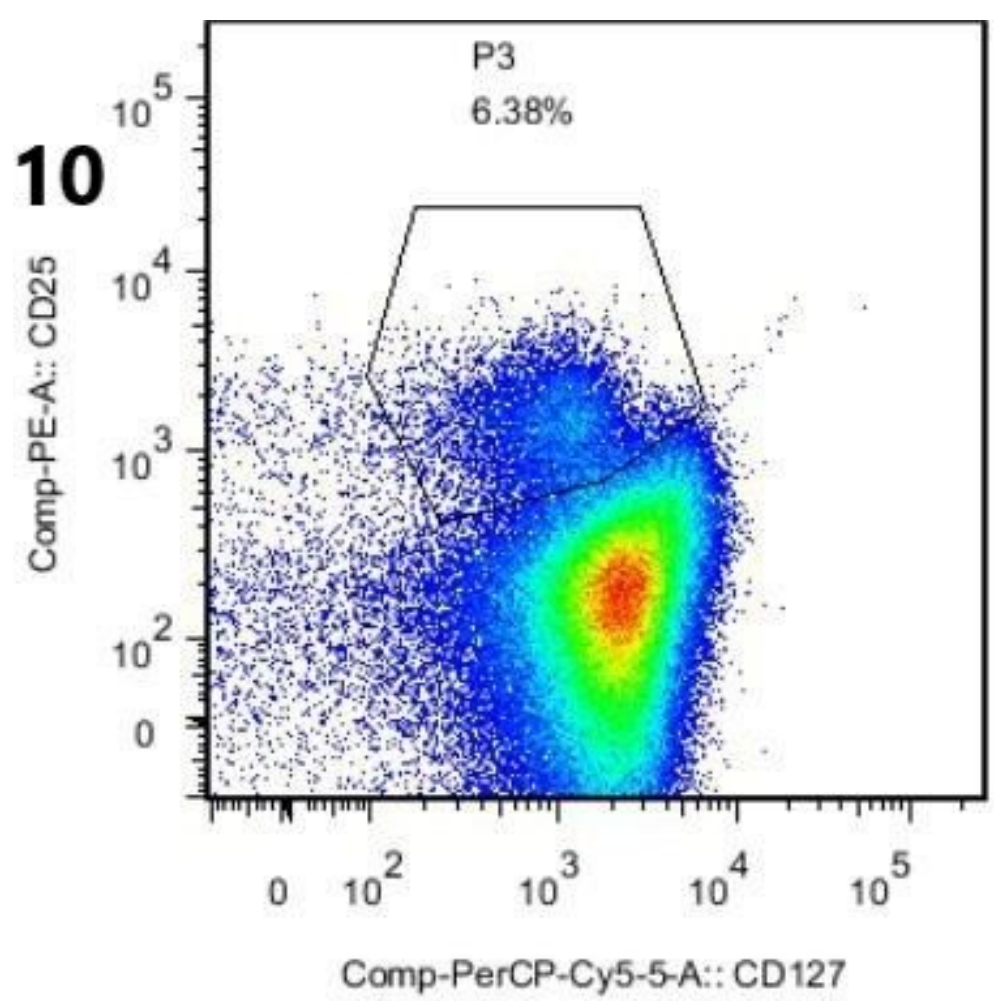

Figure 10

The ratio of CD25+CD127lowT cells to CD4+T cells (GD patients)

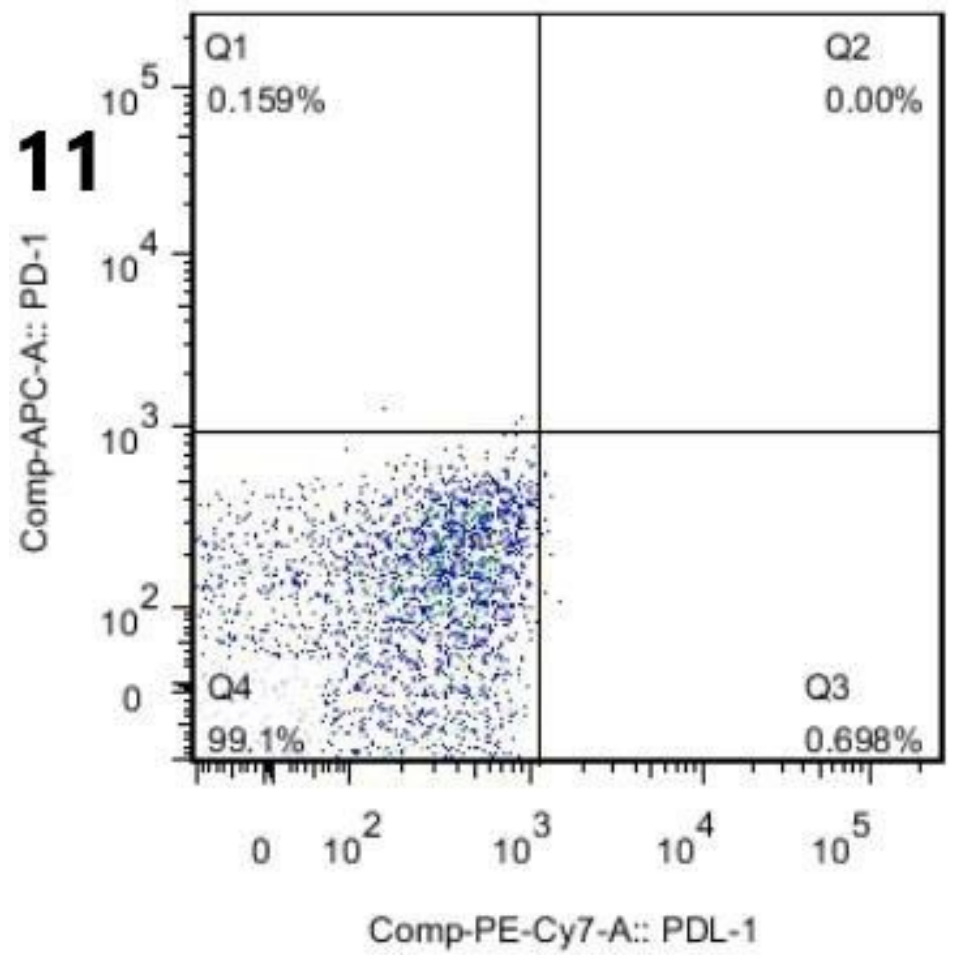

Figure 11

The ratio of PD-1+Treg cells, PD-L1+Treg cells to CD4+CD25+CD127lowT cells (healthy control group) 


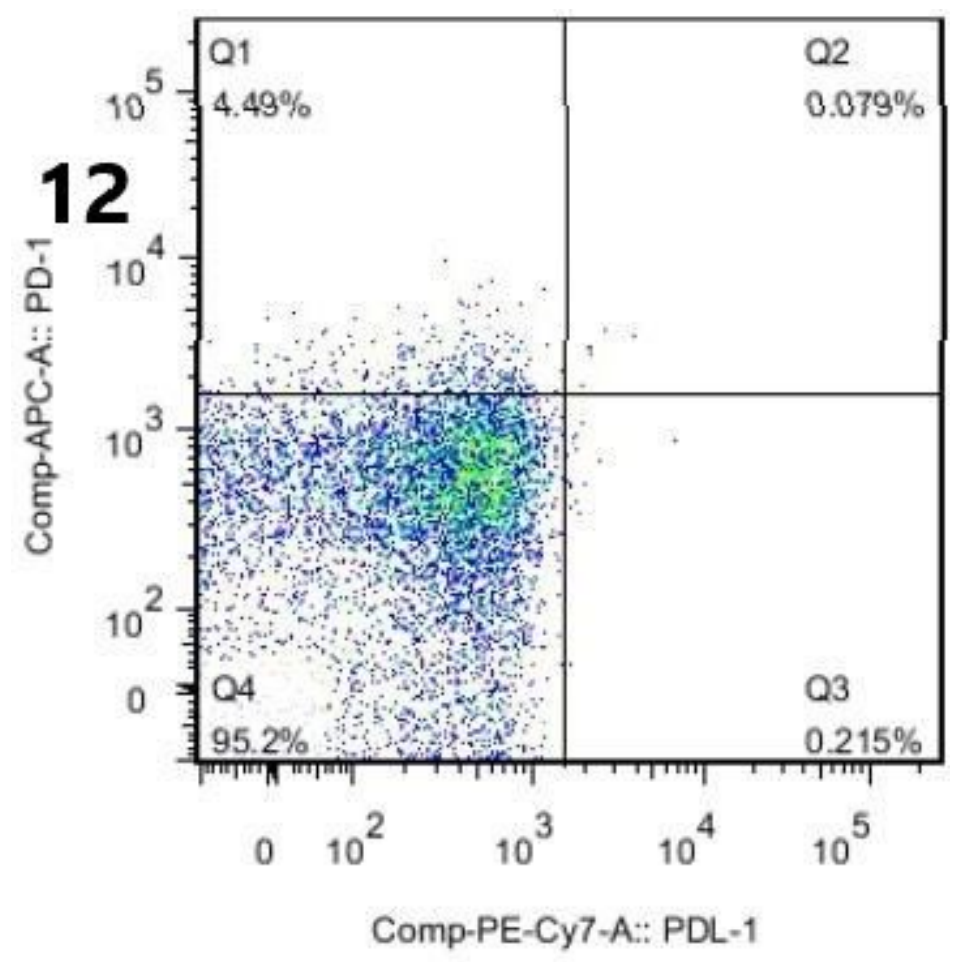

Figure 12

The ratio of PD-1+Treg cells, PD-L1+Treg cells to CD4+CD25+CD127lowT cells (GD patients)

\section{Supplementary Files}

This is a list of supplementary files associated with this preprint. Click to download.

- a.jpg

- b.jpg

- c.jpg 